\title{
Nonexposure laparoscopic and endoscopic cooperative surgery (closed laparoscopic and endoscopic cooperative surgery) for gastric submucosal tumor
}

\author{
Satoru Kikuchi $^{1} \cdot$ Masahiko Nishizaki $^{1} \cdot$ Shinji Kuroda $^{1} \cdot$ Shunsuke Tanabe $^{1} \cdot$ \\ Kazuhiro Noma $^{1}$ - Shunsuke Kagawa ${ }^{1}$ ' Yasuhiro Shirakawa ${ }^{1} \cdot$ Hiroshi Kato $^{2}$ • \\ Hiroyuki Okada ${ }^{3} \cdot$ Toshiyoshi Fujiwara ${ }^{1}$
}

Received: 19 July 2016/Accepted: 29 August 2016/Published online: 6 September 2016

(c) The International Gastric Cancer Association and The Japanese Gastric Cancer Association 2016

\begin{abstract}
Laparoscopic and endoscopic cooperative surgery (LECS) is increasingly applied for gastric submucosal tumors (SMTs) such as gastrointestinal stromal tumors. However, the conventional LECS procedure has the potential risk that gastric contents and even tumor cells could spread into the abdominal cavity because the gastric wall has to be opened during the resection. To avoid this problem, we have developed a modified LECS procedure named "closed LECS." Ten patients underwent closed LECS for the resection of gastric SMTs. Closed LECS consists of the following steps: endoscopic submucosal layer dissection around the tumor, laparoscopic marking of a resection line on the serosal surface along submucosal dissection line, seromuscular suturing with the marked lesion inverted into the inside of the stomach, endoscopic circumferential seromuscular dissection, and peroral retrieval. In three of the initial five cases, the closed LECS procedure was not completed as planned because of the tumor size and endoscopic inappropriate seromuscular dissection. After modification of the procedure, the entire
\end{abstract}

Masahiko Nishizaki

nishi-m3@cc.okayama-u.ac.jp

1 Department of Gastroenterological Surgery, Okayama University Graduate School of Medicine, Dentistry and Pharmaceutical Sciences, 2-5-1 Shikata-cho Kita-ku, Okayama 700-8558, Japan

2 Department of Surgery, Tottori Municipal Hospital, 1-1 Matoba Tottori city, Tottori 680-8501, Japan

3 Department of Gastroenterology and Hepatology, Okayama University Graduate School of Medicine, Dentistry and Pharmaceutical Sciences, 2-5-1 Shikata-cho Kita-ku, Okayama 700-8558, Japan procedure was successful in all five cases. The mean resected tumor diameter was $24.1 \pm 7.6 \mathrm{~mm}$. The mean operation time was $253 \pm 45 \mathrm{~min}$. One patient experienced an intra-abdominal abscess potentially related to delayed perforation as a postoperative complication. The closed LECS procedure for gastric SMTs can theoretically be applied without contamination and tumor cell dissemination into the abdominal cavity.

Keywords Submucosal tumor - Endoscopic submucosal dissection · Laparoscopic gastrectomy · Gastrointestinal stromal tumor

\section{Introduction}

Laparoscopic and endoscopic cooperative surgery (LECS) for gastric submucosal tumor (SMT) was first reported in 2008 by Hiki et al. [1], and its use has rapidly increased recently [2-6]. However, the conventional LECS procedure has the potential risk of gastric contents or tumor cells spilling into the abdominal cavity because the gastric wall has to be opened during the procedure. Therefore, it is thought that this procedure cannot be applied to lesions exposed to the surface of the mucosa such as ulcerated SMT or early gastric cancer.

To avoid this problem, some modified LECS procedures have been investigated, such as inverted LECS [7], a combination of laparoscopic and endoscopic approaches to neoplasia with a nonexposure technique (CLEAN-NET) [8], nonexposed endoscopic wall-inversion surgery (NEWS) [9-13], and endoscopic full-thickness resection [14]. Here we introduce a small series of patients with gastric SMT treated by a novel nonexposure technique, termed "closed LECS." 


\section{Patients and methods}

\section{Patients}

Between June 2013 and April 2016, closed LECS for excision of gastric SMT was performed in ten patients at Okayama University Hospital, Okayama, Japan. The study protocol was approved by the Ethics Review Board of the university (no. 1604-007).

\section{Procedure}

After preparation for laparoscopic gastrectomy, the tumor location was confirmed by endoscopy (Q260J; Olympus, Tokyo, Japan). Under laparoscopy, the tumor location was confirmed by the pressing of endoscopic forceps against the gastric wall from the mucosal side. The mesogastrium and blood vessels around the tumor were dissected and prepared with laparoscopic coagulation shears. Subsequently, mucosal markings were made at the periphery of the tumor under endoscopy, and $10 \%$ glycerin with a small amount of indigo carmine dye was injected into the submucosal layer. The marked area was cut circumferentially with an ITknife2 electrosurgical knife (KD-611L; Olympus, Tokyo, Japan) by the endoscopic submucosal dissection technique, and submucosal dissection was performed deeper and wider above the proper muscular layer (Fig. 1a).

On the laparoscopic side, serosal marking dots were made by electrocautery corresponding to the submucosal dissection line under guidance by endoscopic light (Fig. 1b). Subsequently, a spongy spacer, Securea ${ }^{\circledR}$ (Hogy Medical, Tokyo, Japan), was put on the serosal surface and fixed at the center of the suture line by the suturing of both sides of the serosa, and seromuscular continuous suturing was performed in a way to bury the spacer with use of 3-0 absorbable barbed suture (Fig. 1c, d). Finally, circumferential seromuscular dissection was performed with a DualKnife electrosurgical knife (KD-650L; Olympus, Tokyo, Japan) and the ITknife2 electrosurgical knife along the submucosal dissection line (Fig. 1e). As the full thickness of the wall was cut, the buried Securea spacer was exposed to the luminal side, which ensures successful resection, and the resected lesion and Securea spacer were retrieved perorally.

\section{Statistics}

Pearson correlation analysis was used to test the relation between the case number and the operation time. A $P$ value less than 0.05 was considered statistically significant. Statistical analysis was performed with JMP 12 (SAS Institute, Cary, NC, USA).

\section{Results}

All ten patients in the study underwent abdominal computed tomography, endoscopy, and endoscopic ultrasonography (EUS) preoperatively. All of the tumors were diagnosed as gastric gastrointestinal stromal tumors by EUS-guided fine needle aspiration. The clinicopathological characteristics of the patients are shown in Table 1. Five of the ten tumors were located in the upper third of the stomach.

The surgical outcomes of closed LECS are shown in Table 2. The mean operation time was $253 \pm 45 \mathrm{~min}$, and the mean blood loss was $18 \pm 55 \mathrm{ml}$. The operation time gradually decreased with the number of cases $(P=0.013$, Pearson $r=-0.745$ ) (Fig. 2). In three of the initial five cases, the closed LECS procedure was not completed as planned. In the first case, the tumor was $38 \mathrm{~mm}$ in maximal diameter, and it could not go through the esophagogastric junction. We therefore needed to modify the procedure to open the gastric wall to retrieve the tumor transabdominally. In the initial five cases, endoscopic snare was used for seromuscular dissection in the final step, but it was difficult to dissect the appropriate line finely, which resulted in an enucleation of the tumor and an intraoperative perforation of the stomach.

After the initial five cases, we introduced a spongy spacer, Securea, to facilitate the endoscopic seromuscular dissection. Additionally, endoscopic dissection devices such as the DualKnife and ITknife2 electrosurgical knives were used for seromuscular dissection to determine the dissection line finely. In the later five cases, the entire procedure was performed successfully.

The postoperative course was uneventful except in one case, in which readmission for a Clavien-Dindo classification grade IIIa complication [15], intra-abdominal abscess, was required. The closed LECS procedure was successful without the gastric wall being opened, the postoperative course was uneventful, and the patient was discharged from the hospital on postoperative day 9. The patient was readmitted with a high temperature and abdominal pain on postoperative day 22, and required percutaneous drainage to treat the abscess. During the median follow-up period of 12 months, none of patients exhibited any symptoms or experienced tumor recurrences on endoscopy and computed tomography examination after surgery.

\section{Discussion}

We have described a novel modified LECS procedure, closed LECS. In three of the initial five cases, the closed LECS procedure was unsuccessful because of tumor size and endoscopic inappropriate seromuscular dissection. The 

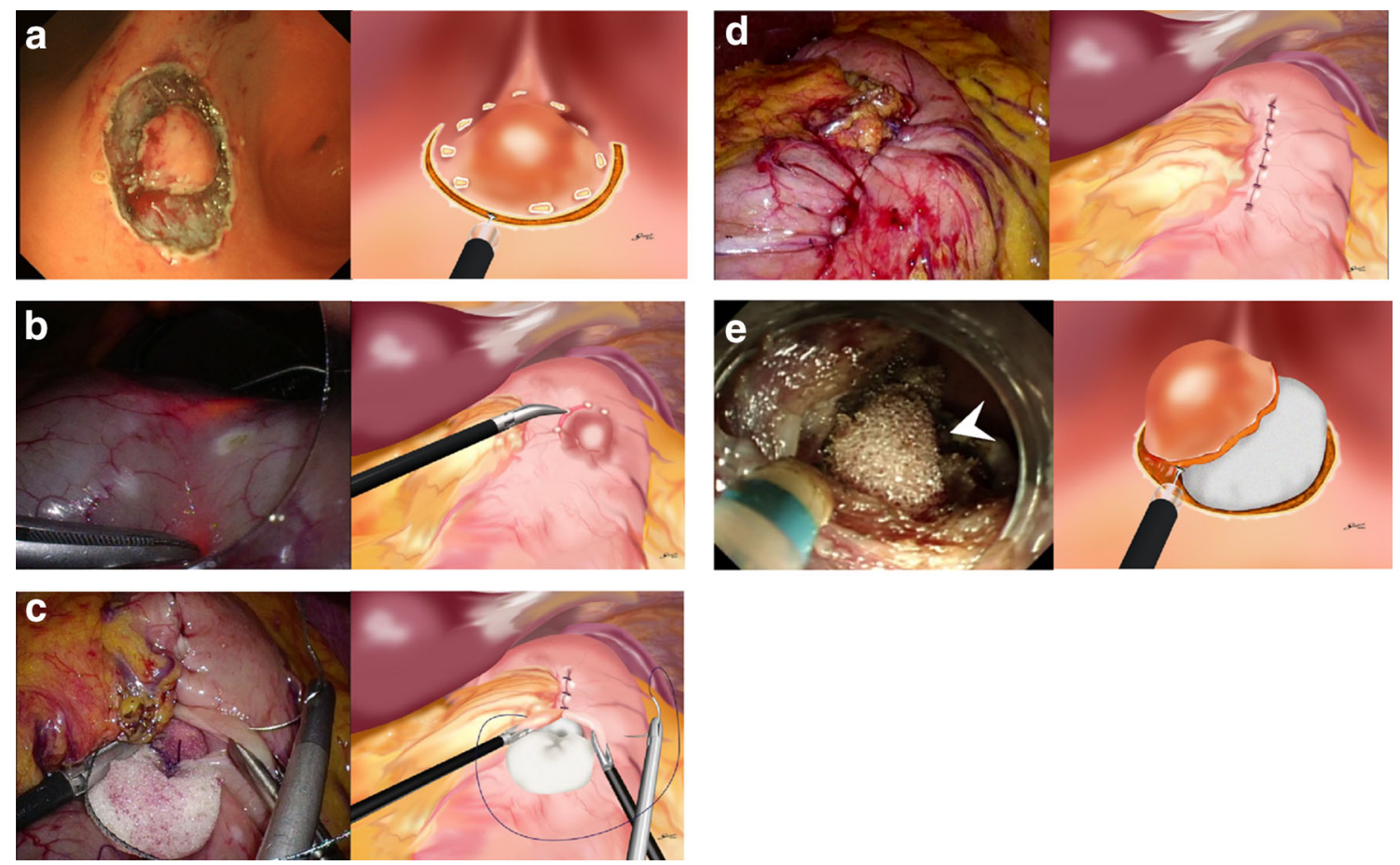

Fig. 1 Procedure for closed laparoscopic and endoscopic cooperative surgery. a Circumferential endoscopic submucosal resection around the tumor by the endoscopic submucosal dissection technique. b Laparoscopic serosal marking on the sides corresponding to the submucosal dissection line under guidance by endoscopic light. c, d Seromuscular suture with inversion of the marked lesion into the

Table 1 Clinicopathological characteristics of the ten patients

\begin{tabular}{ll}
\hline & Values \\
\hline Sex & \\
Male & 5 \\
Female & 5 \\
Age & \\
Mean (years) & $62 \pm 13^{\mathrm{a}}$ \\
Range (years) & $37-75$ \\
Location of tumor & \\
Upper, posterior & 4 \\
Middle, posterior & 1 \\
Upper, lesser curvature & 1 \\
Middle, lesser curvature & 2 \\
Lower, lesser curvature & 2 \\
Tumor size (mm) & $24.1 \pm 7.6^{\mathrm{a}}$
\end{tabular}

${ }^{\mathrm{a}}$ Mean \pm standard error

resected tumor could not go through the esophagogastric junction because the tumor was larger than $35 \mathrm{~mm}$ in case 1. Thus, closed LECS is indicated for gastric SMTs $30 \mathrm{~mm}$ or less in diameter, similar to the indication for the other modified LECS procedures, NEWS and CLEAN-NET. An inverted LECS procedure might be suitable to resect gastric

inside of the stomach in such a way to bury a spongy spacer, Securea ${ }^{\circledR}$ (Hogy Medical, Tokyo, Japan). e Endoscopic seromuscular dissection. The spacer extended the space between the sutured seromuscular plane and the serosal surface of the inverted lesion (arrowhead), and facilitated the seromuscular dissection

Table 2 Surgical outcomes of closed laparoscopic and endoscopic cooperative surgery $(n=10)$

\begin{tabular}{ll}
\hline & Values \\
\hline Operation time (min) & $253 \pm 45^{\mathrm{a}}$ \\
Blood loss (ml) & $18 \pm 55^{\mathrm{a}}$ \\
Unsuccessful execution of procedure & \\
$\quad$ Transabdominal retrieval & 1 \\
$\quad$ Enucleation of tumor & 1 \\
$\quad$ Intraoperative perforation of stomach & 1 \\
Postoperative complications & 1 \\
$\quad$ Intra-abdominal abscess & $9.2 \pm 1.5^{\mathrm{a}}$ \\
Postoperative hospital stay (days) &
\end{tabular}

${ }^{\mathrm{a}}$ Mean \pm standard error

SMTs larger than $30 \mathrm{~mm}$. However, the closed LECS procedure has no limitation with regard to tumor location.

One intraoperative perforation of the stomach and one enucleation of the tumor occurred during the endoscopic seromuscular dissection. The endoscopic view was different from that of the usual endoscopic submucosal dissection, and the appropriate dissection line was threedimensional and difficult to determine in the endoscopic seromuscular dissection. Goto et al. [12] reported that 


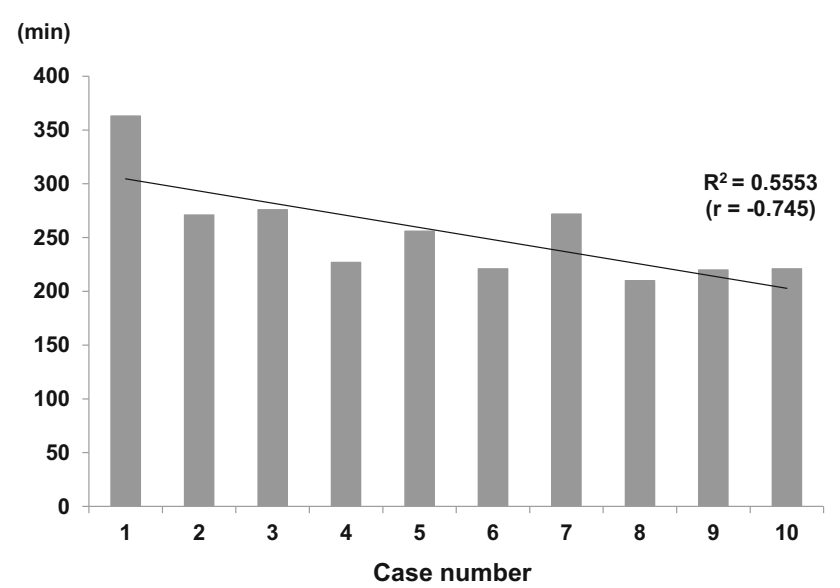

Fig. 2 Surgical outcomes of closed laparoscopic and endoscopic cooperative surgery (closed LECS). The bar graph shows the time required for closed LECS in each case. A trend line was drawn and the coefficient of determination, $R^{2}$, was calculated. $r$ coefficient of correlation

making the space between the sutured seromuscular plane and the serosal surface of the inverted lesion was important. After the initial five cases, we introduced a spongy spacer, which helped to facilitate the endoscopic seromuscular dissection by making the space.

One postoperative complication, intra-abdominal abscess, was observed. It may have been related to the delayed perforation of the anastomotic site by ischemia. To prevent ischemia of the anastomotic site, the dissection of blood vessels around the tumor should be minimized. Endoscopic mucosal suturing with clips might be needed to provide a secure anastomosis such as in NEWS and endoscopic full-thickness resection [9-14].

The concept of closed LECS is similar to that of NEWS [9-13]. However, in the closed LECS procedure, the incision line is determined from the mucosal side; therefore, the appropriate mucosal incision is easy compared with that in NEWS. This indicates closed LECS may be more useful for a condition such as gastric cancer in the future.

There are several limitations to this study. First, this is a preliminary report on a small number of cases, and an additional, larger cohort study is needed to evaluate the procedure. Second, the follow-up period was relatively short to verify whether the tumor recurrence occurred after the operation.

In conclusion, closed LECS is one of the treatment options for small intraluminal-type gastric gastrointestinal stromal tumor without the gastric wall being opened. Closed LECS might have potential to become a minimally invasive surgical procedure for gastric tumors.

\section{Compliance with ethical standards}

Conflict of interest The authors declare that they have no conflict of interest.
Human rights statement and informed consent All procedures followed were in accordance with the ethical standards of the responsible committee on human experimentation (institutional and national) and with the Helsinki Declaration of 1964 and later versions. Informed consent or substitute for it was obtained from all patients for their being included in the study.

\section{References}

1. Hiki N, Yamamoto Y, Fukunaga T, Yamaguchi T, Nunobe S, Tokunaga $\mathrm{M}$, et al. Laparoscopic and endoscopic cooperative surgery for gastrointestinal stromal tumor dissection. Surg Endosc. 2008;22:1729-35.

2. Tsujimoto H, Yaguchi Y, Kumano I, Takahata R, Ono S, Hase K. Successful gastric submucosal tumor resection using laparoscopic and endoscopic cooperative surgery. World J Surg. 2012;36:32730.

3. Kawahira H, Hayashi H, Natsume T, Akai T, Uesato M, Horibe D, et al. Surgical advantages of gastric SMTs by laparoscopy and endoscopy cooperative surgery. Hepatogastroenterology. 2012; 59:415-7.

4. Hara J, Nakajima K, Takahashi T, Yamasaki M, Miyata H, Kurokawa Y, et al. Laparoscopic intragastric surgery revisited: its role for submucosal tumors adjacent to the esophagogastric junction. Surg Laparosc Endosc Percutaneous Tech. 2012;22: 251-4.

5. Hoteya S, Haruta S, Shinohara H, Yamada A, Furuhata T, Yamashita $\mathrm{S}$, et al. Feasibility and safety of laparoscopic and endoscopic cooperative surgery for gastric submucosal tumors, including esophagogastric junction tumors. Dig Endosc. 2014;26:538-44.

6. Hiki N, Nunobe S, Matsuda T, Hirasawa T, Yamamoto Y, Yamaguchi T. Laparoscopic endoscopic cooperative surgery. Dig Endosc. 2015;27:197-204.

7. Nunobe S, Hiki N, Gotoda T, Murao T, Haruma K, Matsumoto H, et al. Successful application of laparoscopic and endoscopic cooperative surgery (LECS) for a lateral-spreading mucosal gastric cancer. Gastric Cancer. 2012;15:338-42.

8. Inoue H, Ikeda H, Hosoya T, Yoshida A, Onimaru M, Suzuki M, et al. Endoscopic mucosal resection, endoscopic submucosal dissection, and beyond: full-layer resection for gastric cancer with nonexposure technique (CLEAN-NET). Surg Oncol Clin N Am. 2012;21:129-40.

9. Mitsui T, Goto O, Shimizu N, Hatao F, Wada I, Niimi K, et al. Novel technique for full-thickness resection of gastric malignancy: feasibility of nonexposed endoscopic wall-inversion surgery (NEWS) in porcine models. Surg Laparosc Endosc Percutaneous Tech. 2013;23:e217-21.

10. Goto O, Mitsui T, Fujishiro M, Wada I, Shimizu N, Seto Y, et al. New method of endoscopic full-thickness resection: a pilot study of non-exposed endoscopic wall-inversion surgery in an ex vivo porcine model. Gastric Cancer. 2011;14:183-7.

11. Mitsui T, Niimi K, Yamashita H, Goto O, Aikou S, Hatao F, et al. Non-exposed endoscopic wall-inversion surgery as a novel partial gastrectomy technique. Gastric Cancer. 2014;17:594-9.

12. Goto O, Takeuchi H, Kawakubo H, Matsuda S, Kato F, Sasaki M, et al. Feasibility of non-exposed endoscopic wall-inversion surgery with sentinel node basin dissection as a new surgical method for early gastric cancer: a porcine survival study. Gastric Cancer. 2015;18:440-5.

13. Goto O, Takeuchi H, Kawakubo H, Sasaki M, Matsuda T, Matsuda S, et al. First case of non-exposed endoscopic wall-inversion surgery with sentinel node basin dissection for early gastric cancer. Gastric Cancer. 2015;18:434-9. 
14. Kim CG, Yoon HM, Cho SJ, Kook MC, Eom BW, Ryu KW, et al. Nonexposure endolaparoscopic full-thickness resection with simple suturing technique. Endoscopy. 2015;47: 1171-4.
15. Dindo D, Demartines N, Clavien PA. Classification of surgical complications: a new proposal with evaluation in a cohort of 6336 patients and results of survey. Ann Surg. 2004;240:20513. 